\title{
Lymphatic and blood vessels in basal and triple-negative breast cancers: characteristics and prognostic significance
}

\author{
Rabab AA Mohammed ${ }^{1,2}$, Ian O Ellis ${ }^{2}$, Ali M Mahmmod ${ }^{1}$, E Claire Hawkes ${ }^{2}$, \\ Andrew R Green ${ }^{2}$, Emad A Rakha ${ }^{3}$ and Stewart G Martin ${ }^{1}$ \\ ${ }^{1}$ Department of Clinical Oncology, School of Molecular Medical Sciences, University of Nottingham, \\ Nottingham, UK; ${ }^{2}$ Histopathology Department, School of Molecular Medical Sciences, University of \\ Nottingham, Nottingham, UK and ${ }^{3}$ Department of Histopathology, Nottingham University Hospitals \\ NHS Trust, Nottingham, UK
}

\begin{abstract}
Basal and triple-negative breast cancer phenotypes are characterised by unfavourable biological behaviour and outcome. Although certain studies have examined their pathological and molecular profile, the vascular characteristics of lymphatic and blood vessels have not been examined. Immunohistochemical staining with podoplanin, CD34 and CD31 was used to examine lymphatic and microvessel density, as well as vascular invasion in 197 basal-like and in 99 triple-negative breast tumours and compared against 200 non-basal and 334 non-triple-negative cases. All specimens were lymph node negative. Vascular invasion was identified as blood or lymphatic vascular invasion by the differential expression of markers. All measurements were correlated with clinicopathological features and prognosis. No significant difference was detected between the basal and triplenegative groups in terms of lymphatic or microvessel density or vascular invasion. However, both the basal and the triple-negative groups showed significantly higher microvessel density than did the non-basal and non-triple-negative groups $(P=0.017$ and $P<0.001$, respectively). Unlike microvessel density, no significant difference was detected in lymphatic density between the basal or triple-negative groups compared with their respective controls. Interestingly, vascular invasion, almost entirely lymphatic invasion, was detected in $27 \%$ of the basal and in $26 \%$ of the triple-negative groups with no significant difference in comparison with control groups. In both basal and triple negatives, vascular invasion was associated with poorer survival by univariate and multivariate analyses. The 20-year overall survival rate in basal-like tumours was $55 \%$ in vascular invasionpositive cases compared with $73 \%$ in vascular invasion-negative tumours $(P=0.012)$, and $46 \%$ in triple-negative vascular invasion-positive compared with $79 \%$ in vascular invasion-negative tumours $(P=0.001)$. Basal-like vs non-basal-like and triple-negative vs non-triple-negative tumours have similar vascular characteristics in terms of lymphatic vessel density and vascular invasion but higher microvessel density, suggesting that such groups may preferentially benefit from anti-angiogenic therapy. Vascular invasion was, in all phenotypes, almost entirely lymphatic vessel invasion and could stratify basal-like and triple-negative phenotypes into distinct prognostic groups.

Modern Pathology (2011) 24, 774-785; doi:10.1038/modpathol.2011.4; published online 4 March 2011
\end{abstract}

Keywords: basal phenotype; breast cancer; lymphatic; lymphovascular invasion; metastasis; triple negative

Correspondence: Dr SG Martin, MSc, PhD, Department of Clinical Oncology, School of Molecular Medical Sciences, Nottingham City Hospital, University of Nottingham, Hucknall Road, Nottingham, NG5 1PB, UK or Dr RAA Mohammed, MBBch, PhD, Faculty of Medicine, Department of Pathology, Assiut University, Assiut, Egypt. E-mail: stewart.martin@nottingham.ac.uk or rabab aamh@yahoo.com

Received 16 July 2010; revised 18 October 2010; accepted 16 November 2010; published online 4 March 2011
Breast cancer of the basal-like phenotype has been a focus of interest in breast cancer research over the last decade. This phenotype was initially described in a study by Perou and colleagues in which DNA microarray technology was used to examine gene expression patterns in primary breast cancers. Five groups were found to have distinct molecular, biological and clinical features. The existence of such types, namely luminal A, luminal B, ERBB2 
(Her-2)-overexpressing, basal-like and normal-like types $^{1,2}$ have also been confirmed using immunohistochemical approaches. ${ }^{3}$

Basal-like breast cancer represents approximately 15-20\% of invasive ductal carcinomas and displays specific molecular, epidemiological, phenotypic, morphological and mammographic features. It is characterised by high histological grade, high growth fraction and distinct genetic alterations. Such tumours are usually negative for hormone receptors and ERBB2 and show high expression of P53, EGFR and KIT. They are more likely to develop distant metastasis and have a generally poorer prognosis. ${ }^{4}$ Their mammographic appearance is also different from that of non-basal tumours; they are more likely to manifest as an ill-defined mass or with comedo calcification, whereas non-basalphenotype tumours are more likely to manifest as a spiculated mass. ${ }^{5}$ There is no one 'gold' standard definition of basal-like breast cancer. It has been defined by negativity for ER, PR and HER2, with positive expression of either basal cytokeratins or EGFR. ${ }^{6}$ In a different report, a positive expression of basal cytokeratins (CK5/6 and/or CK14) was used to identify the basal-like phenotype regardless of the expression of other markers. ${ }^{7}$

The other recently recognised type of breast cancer are triple-negative tumours, which are characterised by being negative for ER, PR and HER2 and which account for $\sim 16 \%$ of breast cancers. ${ }^{7}$ They have distinguishable epidemiological ${ }^{8,9}$ and imaging features. ${ }^{10}$ It was initially believed that both Basallike and triple-negative phenotypes were synonymous; however, recent results have suggested that both types may be distinct. ${ }^{11}$ A comparative analysis of the clinicopathological characteristics and the expression of basal markers found that although triple-negative tumours were significantly associated with the expression of basal markers, $19 \%$ were negative for such markers and $7.3 \%$ of non-triple-negative tumours expressed them, suggesting that the triple-negative phenotype is not an ideal surrogate marker for basal-like breast cancers. ${ }^{12}$ Recent studies have shown that each subtype, basallike and triple-negative, is heterogeneous and consists of subgroups. ${ }^{13}$

Lymph vessels and blood vessels within and around malignant tumours have been known to have an important role in disease progression in many tumour types, including breast cancer. It has been appreciated for some time that angiogenesis (the process of formation of new blood vessels) has an important role in the progression of breast cancer. ${ }^{14-16}$ Angiogenesis is commonly measured by counting blood vessels in tumour sections stained with one of the vascular markers such as CD34 or CD31, yielding microvessel density, and this been found to be associated with features of unfavourable outcome, such as larger size and poor differentiation. However, its role as a prognostic factor in breast cancer was found to be weak. ${ }^{17}$ Much less is known regarding lymph vessels and their role in tumour progression and patient prognosis. The advent of robust markers that allow a clear delineation of lymphatic vessels from blood vessels has allowed lymphatic research to flourish over the last decade. Tumours with high lymph-vessel density have been found to be associated with a higher risk for axillary lymph-node metastasis; ${ }^{18,19}$ however, its role in the prognostication of breast cancer is controversial. In a previous study, from the current authors, on a mixed group of breast cancers (various subtypes and a mix of lymph node-positive and lymph node-negative tumours), high lymph-vessel density was found to be associated with the presence of lymph-node metastasis and shorter overall survival. However, upon multivariate analysis, only tumour grade, lymph-node status and the presence of vascular invasion, but not lymph-vessel density, were found to be independent poor prognostic factors. ${ }^{20}$ The presence of vascular invasion, as detected by standard H\&E methodology, has been well characterised as an indicator of tumour types with higher metastatic potential, greater risk of recurrence and death from the disease, ${ }^{21-23}$ and has been used to guide adjuvant systemic therapy for patients with node-negative breast cancer. ${ }^{24}$ Vascular invasion includes both lymphatic vascular invasion and blood vascular invasion. As mentioned above, historically, it was difficult to distinguish between each type of invasion because of the lack of robust lymphatic-specific markers, but with the discovery of such markers, lymphatic research has expanded. Podoplanin (D2-40) expression can be used to specifically identify lymphatic vessels, ${ }^{25-28}$ and through its use, it has become possible to identify and distinguish lymphatic from blood vascular invasion. ${ }^{29}$ It has been previously reported that basal-like breast cancers are more likely to metastasise to the brain and lung (intra-pulmonary) and that this may be an indication for preferential haematogenous spreading. ${ }^{5}$ Recent results by the current authors have suggested that breast cancer cells show an initial preference for lymphatic rather than for blood vessels. ${ }^{29}$ Such work was conducted on a relatively small cohort of 177 patients, and hence it was not possible to examine whether different breast cancer subtypes showed different patterns of vascular invasion. The current study addresses this by examining vascular invasion patterns in primary basal-like and triple-negative phenotypes by studying a large cohort of lymph node-negative early-stage breast cancers.

The aims of this study were as follows: (1) to examine the vascular characteristics (lymphatic and microvessel density) in breast cancers of basal phenotypes and of triple-negative phenotypes and to compare these characteristics with non-basal tumour and non-triple-negative types; (2) to use immunohistochemical staining methods, with vascular markers, to distinguish between lymphatic and blood vascular invasion, as well as to examine 
the frequency of each type in both tumour groups; and (3) to examine the association between lymphvessel density, microvessel density and vascular invasion with clinicopathological characteristics and patient prognosis.

\section{Materials and methods}

\section{Patients and Tissue Samples}

Clinicopathological data of early-stage 1000 specimens of primary invasive breast cancer were retrieved from the Department of Histopathology (Nottingham University Hospitals, City Hospital Campus). The basal phenotype was identified as specimens expressing CK5/6 and/or CK14 as described previously. ${ }^{7}$ Overall, 197 of 1000 (19.7\%) specimens were found of the basal-like phenotype for which paraffin-embedded archival specimens were retrieved from the Department of Histopathology. A total of 82 patients (42\%) were younger than 50 years. In all, $58(29 \%)$ of the specimens were $<1.5 \mathrm{~cm}$. All specimens were lymph node negative. Overall, $74(38 \%)$ patients had developed a recurrence by the time of the last follow-up and 56 (31\%) had died from breast cancer. To compare the vascular characteristics with non-basal types, 200 consecutive non-basal lymph node-negative primary invasive breast cancer specimens were identified and retrieved from the 1000 cohort. This gave 397 specimens for the current study. More details about the clinicopathological characteristics of the basal and the non-basal groups are summarised in Table 1. From the 397 cohort, 99 tumour specimens were of the triple-negative phenotype (negative ER, negative PR and negative HER2) and 298 were of the nontriple-negative phenotype. A total of $44(44 \%)$ of the triple negatives were from postmenopausal women, $80(80 \%)$ were $>1.5 \mathrm{~cm}$ in size, $88(88 \%)$ were grade III and $8(8 \%)$ were grade II. In all, 26 patients died from the disease, 34 developed a recurrence and 21 (21\%) developed distant metastases. This project was approved by the Nottingham Ethics Committee 2 with 20-year clinical follow-up information available for analysis. The Nottingham Local Research Ethics Committee waived the need for written informed consent.

\section{Immunohistochemistry}

For identification of blood vessels and lymph vessels, representative paraffin-embedded sections from each specimen were stained with CD34 (Serotec, MCAP547, 1:500 dilution) and with podoplanin (AngioBio, 11-003, 1:100 dilution), respectively. In 97 sections, there was CD34 reactivity around tumour cell clusters suggesting the possibility of blood vascular invasion. This was confirmed, or otherwise, by staining parallel sections with CD31 (monoclonal, 0823, DakoCytomation, Denmark,
Table 1 Clinicopathological characteristics of specimens with basal phenotype

\begin{tabular}{|c|c|c|}
\hline & $\begin{array}{c}\text { Basal group } \\
\text { (total no. }=197)\end{array}$ & $\begin{array}{l}\text { Non-basal group } \\
\text { (Total no. }=200)\end{array}$ \\
\hline \multicolumn{3}{|l|}{ Age (years) } \\
\hline$\leq 50$ & $82(42)$ & $57(28)$ \\
\hline$>50$ & $115(58)$ & $143(72)$ \\
\hline \multicolumn{3}{|l|}{ Grade } \\
\hline I & $27(14)$ & $69(34)$ \\
\hline II & 30 (15) & $71(36)$ \\
\hline III & $139(71)$ & $60(30)$ \\
\hline \multicolumn{3}{|l|}{ Recurrence } \\
\hline No & $123(62)$ & $124(64)$ \\
\hline Definite & $74(38)$ & $71(36)$ \\
\hline \multicolumn{3}{|c|}{ Menopausal status } \\
\hline Pre & $88(45)$ & $57(28)$ \\
\hline Post & $109(55)$ & $143(72)$ \\
\hline \multicolumn{3}{|l|}{$P R$} \\
\hline Negative & $136(70)$ & $61(31)$ \\
\hline Positive & $52(26)$ & $112(57)$ \\
\hline Unknown & $9(5)$ & $24(12)$ \\
\hline \multicolumn{3}{|l|}{$D M$} \\
\hline No & $152(78)$ & $159(78)$ \\
\hline Definite & $43(22)$ & 35 (18) \\
\hline \multicolumn{3}{|l|}{ Size $(\mathrm{cm})$} \\
\hline$\leq 1.5$ & $58(29)$ & 99 (49) \\
\hline$>1.5$ & $139(71)$ & $97(51)$ \\
\hline \multicolumn{3}{|l|}{$E R$} \\
\hline Negative & $124(62)$ & $32(16)$ \\
\hline Positive & $65(33)$ & $151(76)$ \\
\hline Unknown & $8(4)$ & $17(8)$ \\
\hline \multicolumn{3}{|l|}{ Death } \\
\hline No & 124 (69) & 139 (69) \\
\hline Yes & $56(31)$ & $46(25)$ \\
\hline
\end{tabular}

DM, distant metastasis; ER, oestrogen receptor; PR, progesterone receptor.

1:100 dilution). In brief, $4-\mu$ m-thick sections were deparaffinised and rehydrated. Antigen retrieval for CD31 and CD34 was achieved by incubating sections in $0.01 \mathrm{~mol} / \mathrm{l}$ sodium citrate buffer ( $\mathrm{pH}$ 6.0) in an $800-\mathrm{W}$ microwave for $20 \mathrm{~min}$. Podoplanin did not require antigen retrieval. After blocking of endogenous hydrogen peroxidase and non-specific reactions, sections were incubated for an hour at room temperature with primary antibodies. Visualisation of staining was conducted using streptABC kit (StreptABComplex/ HRP Duet, Mouse/Rabbit kit, Dako Corporation, K0492) according to the manufacturer's instructions. Immunohistochemical reactions were developed with 3,3'-diaminobenzidine as the chromogenic peroxidase substrate (Dako Corporation, K3468), counterstained with Myer's haematoxylin and mounted.

\section{Assessment of lymph-vessel density and microvessel} density

Lymph-vessel density was assessed in podoplaninstained sections as described previously. ${ }^{20}$ All lymph 

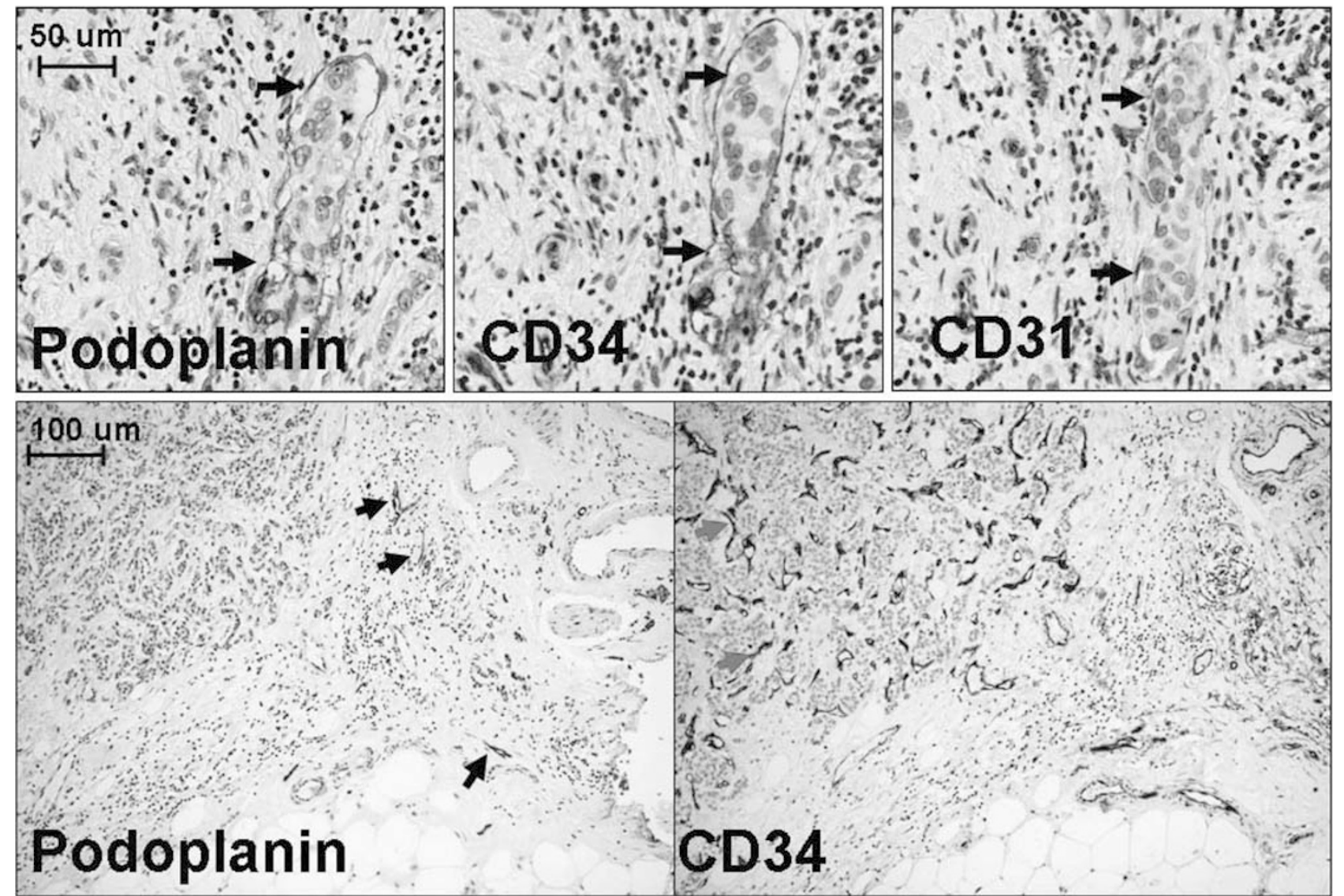

Figure 1 The upper panel shows parallel sections of a breast cancer specimen stained with podoplanin, CD34 and CD31 showing a tumour embolus within a podoplanin-positive, CD34-positive and CD31-positive vessel indicating the lymphatic nature of the vessel, scale bar $=50 \mu \mathrm{m}$. The lower panel shows parallel sections of a breast cancer specimen section stained with podoplanin (showing low lymphatic vessel density, arrows point at lymph vessels) and CD34 (showing high microvessel density, red arrows point to blood vessels), scale $\mathrm{bar}=100 \mu \mathrm{m}$. The colour reproduction of this figure is available on the html full text version of the manuscript.

vessels in the whole tumour section were counted using $\times 100$ magnification with a surface area of $3.46 \mathrm{~mm}^{2}$. The sum of lymph vessels was divided by the sum of the surface area of all counted fields to adjust lymph-vessel density to variation in tumour size. The lymph-vessel density was presented as the number of lymph vessels $/ \mathrm{mm}^{2}$. Microvessel density was assessed in CD34-stained sections using the Chalkley counting method, at $\times 200$ magnification, as described previously. ${ }^{20}$ In brief, the microvessel density count is the average of the count of three vascular hot-spot fields using the Chalkley grid. This method was found to be reliable and reproducible in the assessment of tumour angiogenesis. ${ }^{30,31}$

\section{Assessment of vascular invasion}

Parallel CD34- and podoplanin-stained sections were examined. Where tumour cell clusters were detected within a CD34-positive structure, a further parallel section was stained with CD31 for confirmation of the vascular nature of the lesion (97 section). Vascular invasion lesions were cate- gorised as blood vascular invasion or lymphatic vascular invasion according to the differential expression of the three markers. Tumour clusters within podoplanin ${ }^{+}$were identified as lymphatic vascular invasion regardless of the expression of CD34 or CD31, the expression of which is variable in tumoural lymph vessels (Figure 1). ${ }^{32-34}$ Blood vascular invasion was identified if tumour cells were detected within podoplanin-negative and both CD34- and CD31-positive vessels.

\section{Statistical Analysis}

The following four levels of statistics were performed using SPSS for windows, version 17: (1) Mann-Whitney tests were conducted to compare the means of lymph-vessel density and microvessel density (as continuous data) between the basal and non-basal groups and between the triple-negative and non-triple-negative groups; (2) specimens were divided into two categories according to the median values of lymph-vessel density and microvessel 
density. The association between lymph-vessel density, microvessel density and clinicopathological criteria was evaluated in univariate analysis using a $2 \times 2$ table and $\chi^{2}$ test. (3) Survival analysis of disease-free interval and overall survival was accomplished using the Kaplan-Meier method, and the statistical significance of differences in the cumulative survival curves between groups was evaluated by the long-rank test. Multivariate analysis, using a Cox proportional hazards model, was conducted using patient age, tumour size, tumour grade and vascular invasion. All statistical analyses were two sided with significance defined as $P<0.05$.

\section{Results}

\section{Vascular Characteristics in Basal vs Non-Basal Tumour Types and in Triple-Negative vs Non-Triple-Negative Groups}

\section{Basal-like and non-basal-like tumours}

Clinicopathological characteristics were compared between the basal-like and the non-basal-like groups. Tumours of the basal-like phenotype were characterised by larger tumour size, high grades and negativity for ER and PR. The associations between the clinicopathological characteristics of the basal-like subtypes, prognosis and difference from luminal subtypes have been the subject of many studies and are reported and discussed in detail elsewhere. , $^{3,7,735-37}$

Lymph-vessel density in the total study population ranged between 0.00 and $10.57 / \mathrm{mm}^{2}$, with a mean of $2.2 \pm 0.16$ and a median of 1.43 . The lymphvessel density in the basal group was $2.2 \pm 0.16$ compared with $1.64 \pm 0.14$ in the non-basal group with no significant difference $(P=0.227)$. Microvessel density in the total study population ranged from 1 to 6.7 , with a mean of $2.73 \pm 0.08$ and a median of 2.7. Microvessel density was significantly higher in the basal $(3.73 \pm 0.08)$ than in the non-basal group $(2.60 \pm 0.08)(P=0.017)$. Vascular invasion was detected in $53(27 \%)$ of basal-like specimens and in $21 \%$ of non-basal specimens with no significant difference between the two $(P=0.159$, Table 2). In 50 of the 53 basal-like specimens, invasion was lymphatic vascular invasion, in 2 specimens, the vascular invasion was blood vascular invasion, and in one specimen, there were both lymphatic and blood vascular invasion. In non-basal cases, vascular invasion was detected in 41 of 200 $(21 \%)$ cases, all of which were lymphatic vascular invasion with no blood vascular invasion being detected.

\section{Triple-negative and non-triple-negative tumours} The mean lymph-vessel density in the triplenegative group was $2.01 \pm 0.16$ compared with $1.82 \pm 0.09$ in the triple-negative group, with no significant difference between the two $(P=0.212)$.
Table 2 Vascular characteristics in breast cancers of basal phenotypes vs non-basal types

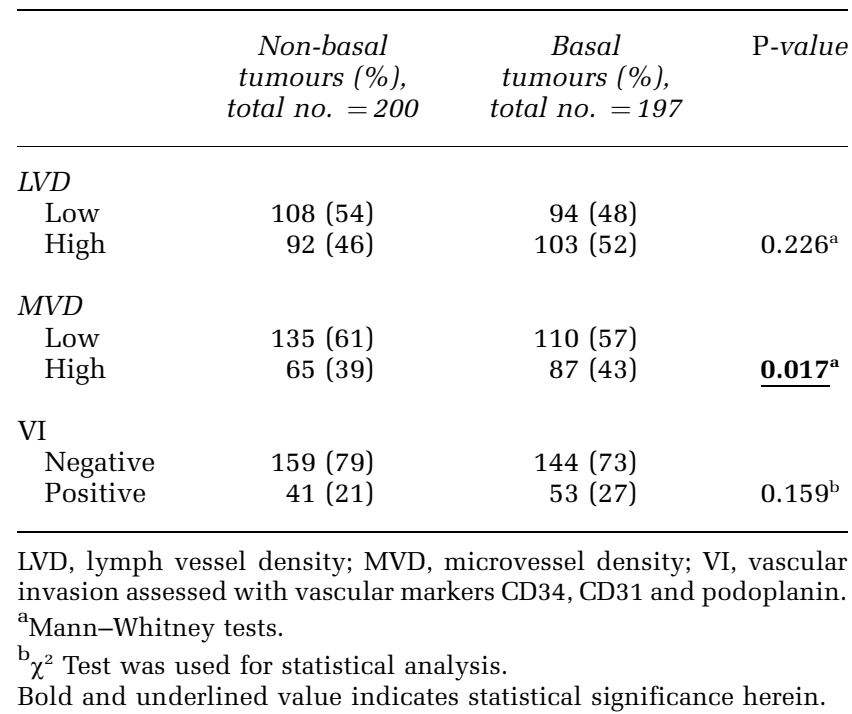

Table 3 Vascular characteristics in breast cancers of triplenegative vs non-triple-negative phenotypes

\begin{tabular}{lccc}
\hline & $\begin{array}{c}\text { Triple-negative } \\
\text { tumours (\%), } \\
\text { total no. }=99\end{array}$ & $\begin{array}{c}\text { Non-triple-negative } \\
\text { tumours (\%), } \\
\text { total no, }=334\end{array}$ & P-value \\
\hline LVD & & & \\
Low & $46(47)$ & $179(54)$ & \\
High & $53(54)$ & $155(46)$ & $0.212^{\text {a }}$ \\
MVD & & & \\
Low & $42(42)$ & $226(68)$ & \\
High & $57(58)$ & $108(32)$ & \\
VI & & & \\
Negative & $73(74)$ & $261(78)$ & 0.359 \\
Positive & $26(26)$ & $73(22)$ & \\
\hline
\end{tabular}

LVD, lymph vessel density; MVD, microvessel density; VI, vascular invasion assessed with vascular markers CD34, CD31 and podoplanin.

${ }^{\mathrm{a}}$ Mann-Whitney tests were used for statistical analysis.

Bold and underlined value indicates statistical significance herein.

Microvessel density was significantly higher in the triple-negative group $(3.1 \pm 0.12)$ than in the NT group $(2.58 \pm 0.06)(P<0.001$, Table 3$)$. Vascular invasion was detected in 26 specimens in the triplenegative group, 24 were lymphatic vascular invasion (without blood vascular invasion) and 2 were blood vascular invasion (without lymphatic vascular invasion). Vascular invasion was detected in 73 of $334(22 \%)$ specimens in the non-triple-negative group; 72 of 73 were lymphatic vascular invasion and 1 of 73 was lymphatic vascular invasion with associated blood vascular invasion. The frequency of vascular invasion was higher in the triplenegative group $(26 \%)$ than $(22 \%)$ in the non-triplenegative group; however, this was not statistically significant $(P=0.359)$. 
Lymph-Vessel Density, Microvessel Density and Vascular Invasion in Basal and Triple-Negative Phenotypes and their Association with Clinicopathological Characteristics

Basal-like tumours

Basal specimens were categorised into two groups according to the median lymph-vessel density (1.43). There were no significant associations with tumour size, grade or hormonal status or with any of the clinicopathological criteria. Specimens were also categorised into two groups based on the median microvessel density (2.7), 87 (43\%) falling into the high microvessel-density category. Such specimens were significantly associated with tumour sizes $>1.5 \mathrm{~cm}(P=0.001)$, poor differentiation (high grades) $(P<0.001)$, negative ER $(P<0.001)$ and negative $\mathrm{PR}$ status $(P=0.008)$. No significant association was detected in relation to recurrence $(P=0.138)$, distant metastasis $(P=0.129)$ or death from the disease $(P=0.191$, Table 4$)$.

Table 4 Association between the presence of MVD and clinicopathological characteristics in breast cancers with basal phenotypes

\begin{tabular}{|c|c|c|c|c|}
\hline & \multicolumn{4}{|c|}{ MVD nо. (\%) } \\
\hline & Low & High & Total & $\mathrm{P}$-value \\
\hline \multicolumn{5}{|l|}{ Age (years) } \\
\hline$\leq 50$ & $38(34)$ & $44(52)$ & 82 & \\
\hline$>50$ & 74 (66) & $41(48)$ & 115 & 0.012 \\
\hline \multicolumn{5}{|c|}{ Menopausal status } \\
\hline Pre & 48 (39) & $40(53)$ & 88 & \\
\hline Post & $64(62)$ & $45(47)$ & 109 & 0.073 \\
\hline \multicolumn{5}{|l|}{ Size $(\mathrm{cm})$} \\
\hline$\leq 1.5$ & 44 (39) & 14 (17) & 58 & \\
\hline$>1.5$ & $68(61)$ & $71(84)$ & 139 & $\underline{0.001}$ \\
\hline \multicolumn{5}{|l|}{ Grade } \\
\hline I & $24(22)$ & $3(4)$ & 27 & \\
\hline II & $24(21)$ & $6(7)$ & 30 & \\
\hline III & $63(45)$ & 76 (89) & 139 & $\leq \mathbf{0 . 0 0 0 1}$ \\
\hline \multicolumn{5}{|l|}{ Recurrence } \\
\hline No & 72 (64) & $51(60)$ & 123 & \\
\hline Definite & $40(36)$ & $34(40)$ & 74 & 0.538 \\
\hline \multicolumn{5}{|l|}{$E R$} \\
\hline Negative & $62(55)$ & $62(73)$ & 124 & \\
\hline Positive & $49(44)$ & 16 (19) & 65 & $\leq \mathbf{0 . 0 0 0 1}$ \\
\hline \multicolumn{5}{|l|}{$P R$} \\
\hline Negative & 67 (59) & 69 (81) & 136 & \\
\hline Positive & $37(36)$ & $12(15)$ & 52 & $\underline{0.003}$ \\
\hline \multicolumn{5}{|l|}{$D M$} \\
\hline No & $91(82)$ & $61(73)$ & 152 & \\
\hline Definite & $20(18)$ & $23(27)$ & 43 & 0.118 \\
\hline \multicolumn{5}{|l|}{ Death } \\
\hline No & 77 (74) & $47(62)$ & 124 & \\
\hline Yes & $27(26)$ & $29(38)$ & 56 & 0.081 \\
\hline
\end{tabular}

Underlined bold figures indicate statistical significance herein.
The presence of vascular invasion (both lymphatic and blood vascular invasion) was significantly associated with tumour sizes $>1.5 \mathrm{~cm}(P=0.001)$, ER-negative specimens $(P=0.002)$, development of recurrence $(P=0.045)$, distant metastasis $(P=0.021)$ and death from the disease $(P=0.016$, Table 5).

A study for correlations between high lymph-vessel density, high microvessel density and the presence of vascular invasion was conducted to determine whether there were any relationships between the three vascular characteristics in basal-like samples. High lymph-vessel density was not associated with high microvessel density $(P=0.116)$, but was significantly associated with the presence of vascular invasion $(P<0.0001)$. No significant association was found between high microvessel density and the presence of vascular invasion $(P=0.533)$.

\section{Triple-negative tumours}

The same analysis was repeated with the triplenegative group, neither lymph-vessel density,

Table 5 Association between the presence of VI and clinicopathological characteristics in breast cancers with basal phenotypes

\begin{tabular}{|c|c|c|c|c|}
\hline & \multicolumn{4}{|c|}{ Presence of VI no. (\%) } \\
\hline & Absent & Present & Total & $\mathrm{P}$-value \\
\hline \multicolumn{5}{|l|}{ Age (years) } \\
\hline$\leq 50$ & $62(43)$ & $20(37)$ & 82 & \\
\hline$>50$ & $83(50)$ & $32(63)$ & 115 & 0.488 \\
\hline \multicolumn{5}{|c|}{ Menopausal status } \\
\hline Pre & $65(45)$ & $23(43)$ & 88 & \\
\hline Post & $79(55)$ & $30(57)$ & 109 & 0.827 \\
\hline \multicolumn{5}{|l|}{ Size $(\mathrm{cm})$} \\
\hline$\leq 1.5$ & $50(34)$ & $8(15)$ & 58 & \\
\hline$>1.5$ & $94(65)$ & $45(85)$ & 139 & $\underline{0.007}$ \\
\hline \multicolumn{5}{|l|}{ Grade } \\
\hline I & 24 (17) & $3(6)$ & 27 & \\
\hline II & 23 (16) & 7 (13) & 30 & \\
\hline III & $96(67)$ & $43(81)$ & 139 & 0.088 \\
\hline \multicolumn{5}{|l|}{ Recurrence } \\
\hline No & $101(70)$ & $29(54)$ & 123 & \\
\hline Definite & $46(30)$ & $28(47)$ & 74 & $\underline{0.045}$ \\
\hline \multicolumn{5}{|l|}{$E R$} \\
\hline Negative & $87(60)$ & $37(70)$ & 124 & \\
\hline Positive & $52(36)$ & $13(24)$ & 65 & 0.271 \\
\hline \multicolumn{5}{|l|}{$P R$} \\
\hline Negative & $96(66)$ & $40(76)$ & 136 & \\
\hline Positive & $42(29)$ & 10 (19) & 52 & 0.335 \\
\hline Total & & & 165 & \\
\hline \multicolumn{5}{|l|}{$D M$} \\
\hline No & $116(82)$ & $36(68)$ & 152 & \\
\hline Definite & $26(18)$ & $17(32)$ & 43 & $\underline{0.021}$ \\
\hline \multicolumn{5}{|l|}{ Death } \\
\hline No & $97(74)$ & $27(55)$ & 124 & \\
\hline Yes & $34(26)$ & $22(45)$ & 56 & 0.016 \\
\hline
\end{tabular}

Bold and underlined values indicate statistical significance herein. 
microvessel density nor vascular invasion was associated with any clinicopathological features, patient age, tumour size or tumour grade.

\section{Survival Analysis of Lymph-Vessel Density, Microvessel Density and Vascular Invasion in Basal-Like and Triple-Negative Groups}

\section{Basal-like tumours}

High lymph-vessel density, high microvessel density and the presence of vascular invasion were analysed with 20-year follow-up data using the log-rank test and Kaplan-Meier survival curves. Neither high lymph-vessel density nor high microvessel density was associated with overall survival $(P=0.343$ and $P=0.133$, respectively) or with disease-free interval $(P=0.628$ and $P=0.181$, respectively).

However, vascular invasion was significantly associated with reduced overall survival and shorter disease-free interval (Figure 2). The 20-year overall survival rate was $55 \%$ in specimens having vascular invasion compared with $73 \%$ in specimens negative for vascular invasion $(P=0.012)$. Similarly, the 20-year disease-free interval was $25 \%$ with the presence of vascular invasion compared with $49 \%$ in the absence of vascular invasion $(P=0.046$, Table 6$)$. In multivariate analysis of vascular invasion for overall survival, tumour grade $(P=0.01)$ and vascular invasion $(P=0.044)$ retained significance. Tumour size and oestrogen receptor status were of borderline significance $(P=0.050$ and $P=0.055$, respectively, Table 7). Multivariate analysis for disease-free interval using the same variables showed no significance.

\section{Triple-negative tumours}

The same analyses, as described above, were conducted on the triple-negative group. Similar to

Table 6 Univariate survival analysis of VI, MVD and LVD in breast cancers with basal phenotypes

\begin{tabular}{|c|c|c|c|c|c|c|}
\hline & \multicolumn{3}{|c|}{ With overall survival } & \multicolumn{3}{|c|}{ With disease-free interval } \\
\hline & $\begin{array}{c}\text { 20-Year } \\
\text { overall } \\
\text { survival (\%) }\end{array}$ & $\chi^{2}$ & P-value & $\begin{array}{c}\text { 20-Year } \\
\text { disease-free } \\
\text { survival (\%) }\end{array}$ & $\chi^{2}$ & P-value \\
\hline \multicolumn{7}{|l|}{$V I$} \\
\hline Negative & 73 & 6.241 & 0.012 & 49 & 3.990 & 0.046 \\
\hline Positive & 55 & & & 25 & & \\
\hline \multicolumn{7}{|l|}{$M V D$} \\
\hline Low & 70 & 2.252 & 0.133 & 63 & 1.791 & 0.181 \\
\hline High & 61 & & & 54 & & \\
\hline \multicolumn{7}{|l|}{$L V D$} \\
\hline Low & 72 & 0.899 & 0.343 & 63 & 0.235 & 0.628 \\
\hline High & 66 & & & 59 & & \\
\hline
\end{tabular}

Bold and underlined values indicate statistical significance herein.
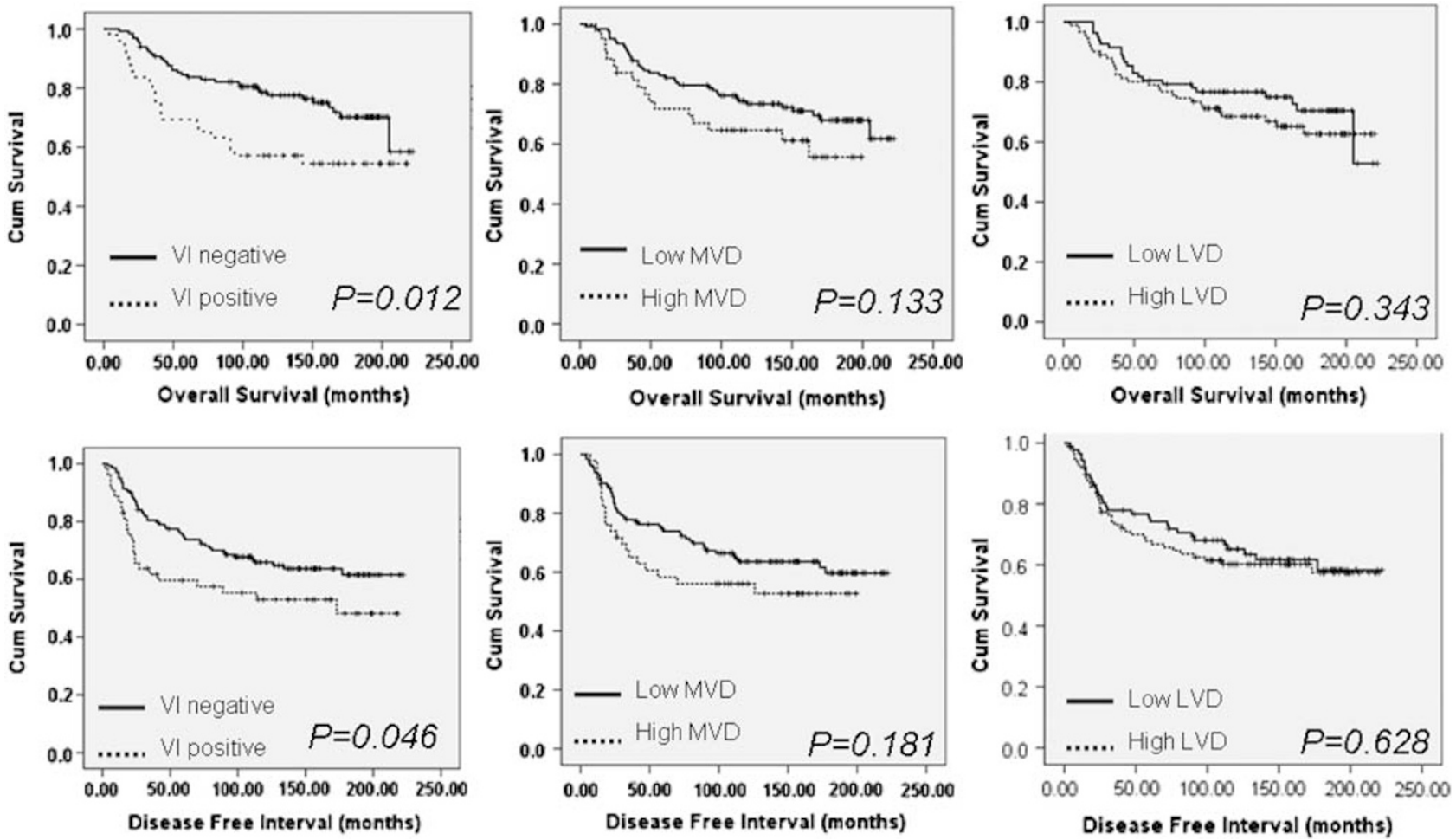

Figure 2 Survival analysis of vascular invasion (VI), microvessel density (MVD) and lymphatic vessel density (LVD) using Kaplan-Meier survival curves in the basal group. The presence of VI is significantly associated with shorter OS $(P=0.012)$ and with shorter disease-free interval $(P=0.046)$. MVD and LVD have no significant associations with either disease-free interval or overall survival. 
Table 7 Multivariate survival analysis of tumour size, grade, VI, ER, PR and HER2 status in relation to overall survival in breast cancers with basal phenotypes (no.=197)

\begin{tabular}{|c|c|c|c|c|c|}
\hline \multirow[t]{2}{*}{ Variables in the equation } & \multirow[t]{2}{*}{$B$} & \multirow[t]{2}{*}{ Sig. } & \multirow[t]{2}{*}{$\operatorname{Exp}(B)$} & \multicolumn{2}{|c|}{$95.0 \%$ CI for $\operatorname{Exp}(B)$} \\
\hline & & & & Lower & Upper \\
\hline $\begin{array}{l}\text { Size }(\leq 1.5 \mathrm{~cm} \text { and }>1.5 \mathrm{~cm}) \\
\text { Grade (I. II and III) }\end{array}$ & 0.726 & $\begin{array}{l}0.050 \\
\mathbf{0 . 0 1 0}\end{array}$ & 2.068 & 0.999 & 4.280 \\
\hline Grade (I) & 1.100 & 0.137 & 3.003 & 0.704 & 12.805 \\
\hline Grade (II) & 1.799 & 0.004 & 6.042 & 1.758 & 20.763 \\
\hline VI (based on vascular markers - ve and +ve) & 0.581 & 0.044 & 1.787 & 1.017 & 3.142 \\
\hline Progesterone receptors status (-ve and +ve) & 0.110 & 0.788 & 1.116 & 0.501 & 2.483 \\
\hline Oestrogen receptors status (-ve and +ve) & 1.414 & 0.055 & 4.113 & 0.972 & 17.409 \\
\hline HER2 & & 0.249 & & & \\
\hline HER2 (1) & -0.523 & 0.287 & 0.593 & 0.226 & 1.554 \\
\hline HER2 (2) & -0.094 & 0.878 & 0.911 & 0.275 & 3.017 \\
\hline HER2 (3) & -1.196 & 0.071 & 0.302 & 0.083 & 1.108 \\
\hline
\end{tabular}

Bold values indicate statistical significance herein.
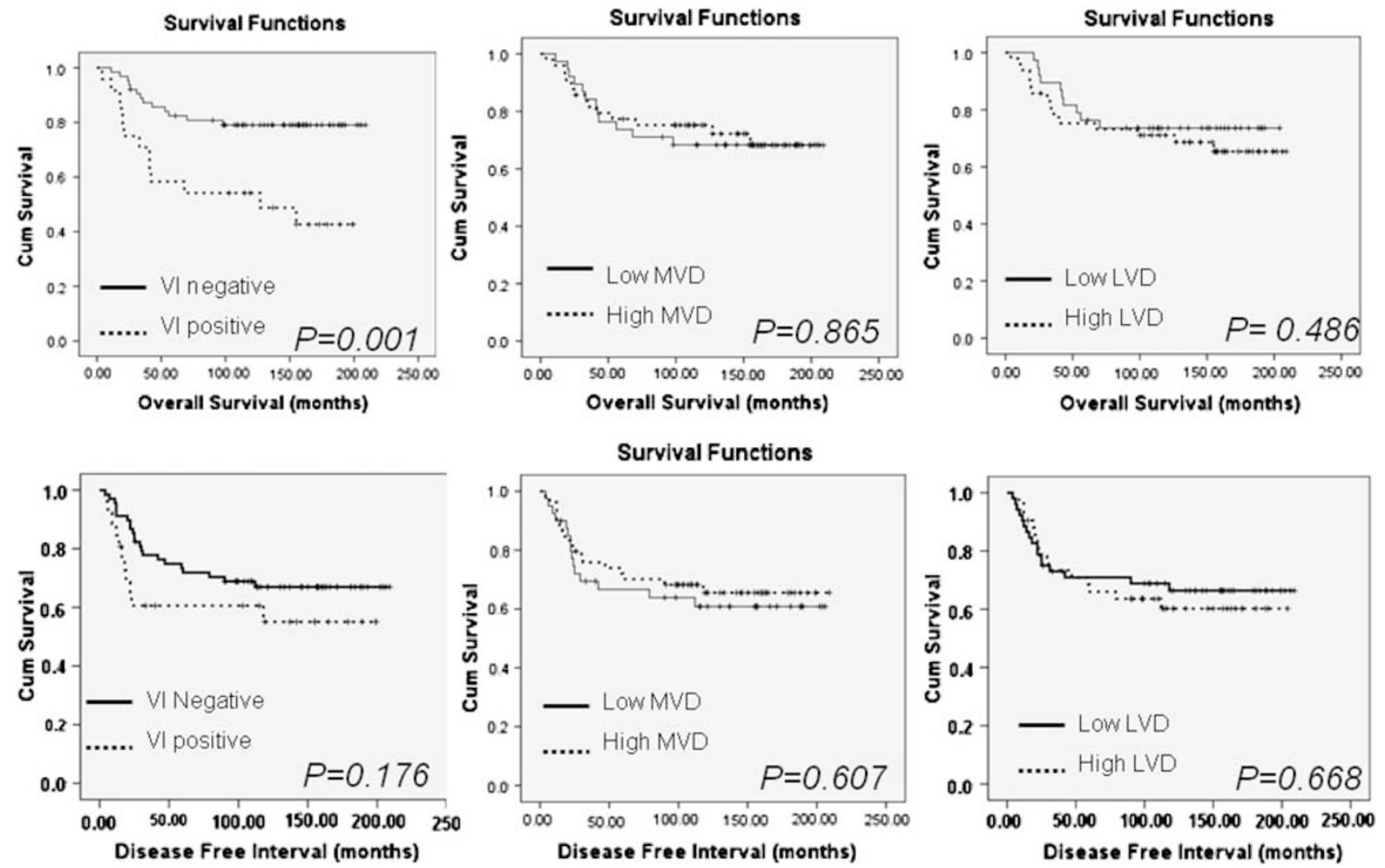

Figure 3 Survival analysis of vascular invasion (VI), microvessel and lymphatic vessel density using Kaplan-Meier survival curves in the triple-negative group. The presence of VI is significantly associated with shorter overall survival $(P=0.001)$ but not with disease-free interval $(P=0.176)$. Microvessel and lymphatic vessel density have no significant associations with either disease-free interval or overall survival.

the basal-like group, neither high lymph-vessel density nor high microvessel density was associated with reduced survival $(P=0.486$ and $P=0.865$, respectively) or with reduced disease-free interval $(P=0.668$ and $P=0.607$, respectively). The presence of vascular invasion was significantly associated with overall survival $(P<0.001)$, but not with disease-free interval $(P=0.176$, Table 8 and Figure 3). In multivariate analysis, vascular invasion retained significance $(P=0.010)$, whereas size and grade did not $(P=0.156$ and $P=0.997$, respectively).

\section{Discussion}

Breast cancers with basal-like and triple-negative phenotypes are recently identified tumour subtypes 
Table 8 Univariate survival analysis of VI, MVD and LVD in breast cancers with TN phenotypes

\begin{tabular}{|c|c|c|c|c|c|c|}
\hline & \multicolumn{3}{|c|}{$\begin{array}{l}\text { With overall } \\
\text { survival }\end{array}$} & \multicolumn{3}{|c|}{$\begin{array}{l}\text { With disease-free } \\
\quad \text { interval }\end{array}$} \\
\hline & $\begin{array}{c}\text { 20-Year } \\
\text { overall } \\
\text { survival (\%) }\end{array}$ & $\chi^{2}$ & P-value & $\begin{array}{c}\text { 20-Year } \\
\text { disease-free } \\
\text { survival (\%) }\end{array}$ & $\chi^{2}$ & P-value \\
\hline \multicolumn{7}{|l|}{$V I$} \\
\hline Negative & 79 & 10.132 & 0.001 & 68 & 1.831 & 0.176 \\
\hline Positive & 46 & & & 58 & & \\
\hline \multicolumn{7}{|l|}{$M V D$} \\
\hline Low & 68 & 0.029 & 0.865 & 62 & 0.264 & 0.607 \\
\hline High & 71 & & & 66 & & \\
\hline \multicolumn{7}{|l|}{$L V D$} \\
\hline Low & 74 & 0.486 & 0.486 & 16 & 0.184 & 0.668 \\
\hline High & 67 & & & 17 & & \\
\hline
\end{tabular}

Bold value indicates statistical significance herein.

characterised by aggressive behaviour and poor prognosis. Tumour lymphatics and blood vessels are known to have an important role in tumour progression in many tumour types, including breast cancer. The aims of this study were to examine the vascular characteristics, lymph-vessel density, microvessel density and vascular invasion, both lymphatic and blood vascular invasion, in basal-like and in triple-negative phenotypes and to study the association of these features with the different clinicopathological criteria and patient prognosis. Previous work has demonstrated that basal-like tumours show a specific pattern of distant metastasis with an increased propensity for visceral metastases to the brain and lung and that they are less likely to metastasise to the bone and liver, suggesting that such tumours might also possess a distinct mechanism of metastatic spread from nonbasal-like tumours. ${ }^{36,37}$ Therefore, it was of interest to examine whether these vascular characteristics differed in basal compared with non-basal tumours and in triple-negative compared with non-triplenegative tumours, which may contribute to the difference in metastatic spread pattern. It was also of interest to investigate whether this difference, if present, contributes to biological and clinical features and, if so, whether they could be used to aid in the determination of prognosis in these two tumour subtypes.

Numerous studies have examined the vascular characteristics of breast cancers; however, no study has previously examined these characteristics, including lymphatics, in basal-like or triplenegative subtypes.

Lymph vessels have, following the characterisation of specific markers, been a focus of attention over the last decade. ${ }^{38,39}$ Similar to previous findings from studies on breast cancer of mixed phenotypes, ${ }^{40,41}$ we found that the majority of lymphatics in the basal phenotype group were located in the peritumoral and the peripheral areas with few tumours having lymphatics within the tumour core. Breast cancers with high lymph-vessel density have been found, in some studies, to be associated with higher risk of lymph-node metastasis and with tumours with negative hormonal-receptor status. However, such associations were not found in other studies. ${ }^{41}$ The significance of high lymph-vessel density as a factor indicating poor prognosis is also controversial. In the study by Kato et al, ${ }^{41}$ lymphvessel density was not associated with overall survival or disease-free interval. In recent studies, from the current authors, high lymph-vessel density was significantly associated with the presence of lymph-node and distant metastases and hence with poorer Nottingham prognostic index and tumour stage. In univariate survival analysis, high lymphvessel density was associated with poorer survival, but this did not retain significance in multivariate analysis. ${ }^{20}$ In the current study, high lymph-vessel density in lymph node-negative basal-like or triplenegative phenotypes was not associated with any of the clinicopathological characteristics or with overall survival or disease-free interval. The difference in such findings may be due to the fact that all current specimens were lymph node negative, whereas those in the previous study were nonselected cases of both lymph node-negative and lymph node-positive specimens. It is generally accepted that lymphatic vessels are a key route for tumour cell dissemination. It is logical to assume that the presence of more vessels may increase the chance of vascular invasion occurring and therefore influence prognosis. When lymph-vessel density in the basal-like was compared with that in the non-basal-like, no difference was found. The same applies to triple-negative and non-triple-negative types. Such data suggest that the density of lymph vessels, itself, may not be of clinical or prognostic significance in either basal-like or triple-negative breast cancer.

As mentioned previously, the clinical and prognostic significance of microvessel density has been examined extensively in breast cancer; however, its use in practice is still fraught with difficulties because of the difference in methods of assessment and the presence of significant inter- and intraobserver variation. ${ }^{42}$ Controversy exists regarding the usefulness of microvessel density as a prognostic factor. ${ }^{17}$ Breast cancers with high microvessel density have, in certain studies, been found to have significant association with larger tumour size, high grade, lymph-node metastasis and poor prognosis; ${ }^{16,18,43,44}$ however, no such association was detected with pathological criteria or patient survival in other studies. ${ }^{45,46}$ In the current study, microvessel density, in both basal-like and triplenegative phenotypes, was significantly higher than in non-basal-like and non-triple-negative types. In basal-like breast cancers, high microvessel density was significantly associated with larger tumour size $(>1.5 \mathrm{~cm})$, high grade, ER negativity and PR 
negativity. Previous studies have reported that basallike breast cancers are generally characterised by being of high grade and of large tumour size. ${ }^{4}$ In the current study, in 197 basal-like breast cancers, 71\% of the tumours were grade III and $71 \%$ were $>1.5 \mathrm{~cm}$ in size, which may explain the association between high microvessel density and basal-like phenotype when compared with non-basal-like tumours. The same was found with triple-negative phenotypes in which the triple negative was associated with high microvessel density when compared with non-triple negative; however, unlike the basal-like phenotype, high microvessel density was not associated with tumour size or grade in the triple-negative group. The same explanation, as in basal-like cancers, can be applied here as the majority of triple-negative cases were of high grade and of large size. Overall, $88 \%$ of tumours in the current cohort of triple-negative specimens were grade III and $80 \%$ were $>1.5 \mathrm{~cm}$. The fact that high microvessel density was not associated with clinicopathological characteristics may be due to the heterogeneous nature of this group. Recently, it was found that triple-negative breast cancers encompass a biologically heterogeneous group of tumours with a proportion of them expressing basal markers. ${ }^{13}$

From survival analyses, microvessel density was not associated with either overall survival or disease-free interval, in basal-like or in triplenegative tumours. Such data suggest that although blood vessels are important for tumour growth and development, they have a little part in the initial dissemination of cancer. It could be hypothesised that such groups may preferentially benefit from anti-angiogenic therapy in terms of primary tumour response. Lymphatic vascular invasion data would suggest, however, that there would be little impact on metastatic dissemination and overall survival. It is interesting, in this light, to reflect on recent work that reports anti-angiogenic therapies reducing primary tumour growth and microvessel density but accelerating invasion and metastasis. ${ }^{47,48}$

The current study, as with our and others' previous findings, ${ }^{20,27,40}$ shows that blood vascular and lymphatic characteristics do not correlate, in either phenotype, in that specimens having high microvessel density do not necessarily have high lymph-vessel density and vice versa, suggesting distinct biological regulatory mechanisms.

Vascular invasion, including both lymphatic and blood vascular invasion, has been generally accepted, for some time, as a risk factor for development of recurrence and death from the disease. With the development of specific blood and lymphatic vascular markers, it has become possible to identify and distinguish lymphatic and blood vascular invasion. In a previous study, the current authors found that, in a cohort of 177 non-selected cases, vascular invasion in breast cancer was almost entirely lymphatic vascular invasion; ${ }^{29}$ such results are currently being validated using 1000 lymph node-negative specimens. Basal-like breast cancers have been reported to show preferable metastatic dissemination to sites such as the brain and liver, and less to the bone. ${ }^{5,49,50}$ These distinctive metastatic patterns have been interpreted as a distinctive ability of basal-like breast cancers for early blood-borne metastasis. It was assumed, from such observations, that the frequency of blood vascular invasion would be higher in basal-like than in the non-basal-like group. Vascular invasion was detected in $27 \%$ of the basal-like tumours compared with $21 \%$ in the non-basal-like group and was $26 \%$ in the triple-negative group compared with $22 \%$ in the non-triple-negative group. In both basal-like and triple-negative cancers, vascular invasion was, interestingly, almost entirely invasion of lymph vessels. Such findings show that both triple-negative and basal-like breast cancers are no different, in terms of initial route of dissemination, from non-basal-like or non-triple-negative types in that such dissemination occurs through lymph vessels and not blood vessels. The distinct metastatic pattern mentioned earlier, to the brain and lung, may be due to other pathomolecular features in basal-like breast cancers and, perhaps, due to other tissue-specific factors in the remote sites themselves that attract basal-like cancer cells to extravasate and to grow. The haematogenous spread of breast cancer cells may occur distal to the primary tumour.

It was interesting to find that the presence of vascular invasion stratified patients, in both basallike and in triple-negative phenotypes, into separate prognostic groups. Such findings emphasise the role of vascular invasion as an independent poor prognostic factor in both tumour phenotypes. Additional studies are required to explore the pathomolecular mechanisms that underlie the highly invasive nature of basal-like breast cancers and the mechanisms of the reported haematogenous metastatic pattern of this subgroup. Such understanding is essential for the optimal management of patients with breast cancer and for finding the appropriate tailored therapy for each patient.

In conclusion, although basal-like and triplenegative breast cancers have been reported, haematogenous patterns of metastatic spread vascular invasion was, with both groups, almost entirely invasion of lymph vessels. Results show that both triple-negative and basal-like cancers are no different, in terms of the initial route of dissemination, from non-basal-like or non-triple-negative types in that such dissemination occurs through lymph vessels and not blood vessels. Similarly, lymph vessel-density in breast cancer with basal or triplenegative phenotypes was no different from those in non-basal or non-triple-negative groups. Basal-like and triple-negative tumours did, however, exhibit higher microvessel density, a finding that may have implications for the use of anti-angiogenic therapy, for local control, in both tumour phenotypes. 
The presence of vascular invasion is an independent poor prognostic factor for overall survival and can be used to stratify basal-like and triple-negative phenotypes into distinct subgroups.

\section{Acknowledgements}

We gratefully acknowledge the Egyptian Government, Ministry of Higher Education (Assiut University, Faculty of Medicine, QAU) and Cancer Research UK for funding this work (grant number C20420/A9967).

\section{Disclosure/conflict of interest}

The authors declare no conflict of interest.

\section{References}

1 Perou CM, Sørlie T, Eisen MB, et al. Molecular portraits of human breast tumours. Nature 2000; 406:747-752.

2 Sørlie T, Perou CM, Tibshirani R, et al. Gene expression patterns of breast carcinomas distinguish tumor subclasses with clinical implications. Proc Natl Acad Sci USA 2001;98:10869-10874.

3 Rakha EA, El-Rehim DA, Paish C, et al. Basal phenotype identifies a poor prognostic subgroup of breast cancer of clinical importance. Eur J Cancer 2006;42: 3149-3156.

4 Rakha EA, Putti TC, Abd El-Rehim DM, et al. Morphological and immunophenotypic analysis of breast carcinomas with basal and myoepithelial differentiation. J Pathol 2006;208:495-506.

5 Luck AA, Evans AJ, Green AR, et al. The influence of basal phenotype on the metastatic pattern of breast cancer. Clin Oncol (R Coll Radiol) 2008;20:40-45.

6 Nielsen TO, Hsu FD, Jensen K, et al. Immunohistochemical and clinical characterization of the basal-like subtype of invasive breast carcinoma. Clin Cancer Res 2004;10:5367-5374.

7 Rakha EA, El-Sayed ME, Green AR, et al. Breast carcinoma with basal differentiation: a proposal for pathology definition based on basal cytokeratin expression. Histopathology 2007;50:434-438.

8 Dolle JM, Daling JR, White E, et al. Risk factors for triple-negative breast cancer in women under the age of 45 years. Cancer Epidemiol Biomarkers Prev 2009; 18:1157-1166.

9 Trivers KF, Lund MJ, Porter PL, et al. The epidemiology of triple-negative breast cancer, including race. Cancer Causes Control 2009;20:1071-1082.

10 Uematsu T, Kasami M, Yuen S. Triple-negative breast cancer: correlation between MR imaging and pathologic findings. Radiology 2009;250:638-647.

11 Bertucci F, Finetti P, Cervera N, et al. How basal are triple-negative breast cancers? Int J Cancer 2008;123: 236-240.

12 Tan DS, Marchió C, Jones RL, et al. Triple negative breast cancer: molecular profiling and prognostic impact in adjuvant anthracycline-treated patients. Breast Cancer Res Treat 2008;111:27-44.
13 Rakha EA, Elsheikh SE, Aleskandarany MA, et al. Triple-negative breast cancer: distinguishing between basal and nonbasal subtypes. Clin Cancer Res 2009;15:2302-2310.

14 Weidner N, Semple JP, Welch WR, et al. Tumor angiogenesis and metastasis-correlation in invasive breast carcinoma. N Engl J Med 1991;324:1-8.

15 Toi M, Kashitani J, Tominaga T. Tumor angiogenesis is an independent prognostic indicator in primary breast carcinoma. Int J Cancer 1993;55:371-374.

16 Bevilacqua $\mathrm{P}$, Barbareschi $\mathrm{M}$, Verderio $\mathrm{P}$, et al. Prognostic value of intratumoral microvessel density, a measure of tumor angiogenesis, in node-negative breast carcinoma-results of a multiparametric study. Breast Cancer Res Treat 1995;36:205-217.

17 Des Guetz G, Uzzan B, Nicolas P, et al. Microvessel density as a prognostic factor in women with breast cancer: a systematic review of the literature and metaanalysis. Cancer Res 2004;64:2941-2955.

18 Choi WW, Lewis MM, Lawson D, et al. Angiogenic and lymphangiogenic microvessel density in breast carcinoma: correlation with clinicopathologic parameters and VEGF-family gene expression. Mod Pathol 2005;18:143-152.

19 Nakamura Y, Yasuoka H, Tsujimoto M, et al. Lymph vessel density correlates with nodal status, VEGF-C expression, and prognosis in breast cancer. Breast Cancer Res Treat 2005;91:125-132.

20 Mohammed RA, Ellis IO, Elsheikh S, et al. Lymphatic and angiogenic characteristics in breast cancer: morphometric analysis and prognostic implications. Breast Cancer Res Treat 2009;113:261-273.

21 Pinder SE, Ellis IO, Galea M, et al. Pathological prognostic factors in breast cancer. III. Vascular invasion: relationship with recurrence and survival in a large study with long-term follow-up. Histopathology 1994;24:41-47.

22 Gajdos C, Tartter PI, Bleiweiss IJ. Lymphatic invasion, tumor size, and age are independent predictors of axillary lymph node metastases in women with T1 breast cancers. Ann Surg 1999;230:692-696.

23 Lee AH, Pinder SE, Macmillan RD, et al. Prognostic value of lymphovascular invasion in women with lymph node negative invasive breast carcinoma. Eur J Cancer 2006;42:357-362.

24 Goldhirsch A, Wood WC, Gelber RD, et al. Meeting highlights: updated international expert consensus on the primary therapy of early breast cancer. J Clin Oncol 2003;21:3357-3365.

25 Breiteneder-Geleff S, Soleiman A, Horvat R, et al. Podoplanin-a specific marker for lymphatic endothelium expressed in angiosarcoma. Verh Dtsch Ges Pathol 1999;83:270-275.

26 Birner P, Obermair A, Schindl M, et al. Selective immunohistochemical staining of blood and lymphatic vessels reveals independent prognostic influence of blood and lymphatic vessel invasion in early-stage cervical cancer. Clin Cancer Res 2001;7:93-97.

27 Schoppmann SF, Birner P, Studer P, et al. Lymphatic microvessel density and lymphovascular invasion assessed by anti-podoplanin immunostaining in human breast cancer. Anticancer Res 2001;21: 2351-2355.

28 Evangelou E, Kyzas PA, Trikalinos TA. Comparison of the diagnostic accuracy of lymphatic endothelium markers: Bayesian approach. Mod Pathol 2005;18: 1490-1497. 
29 Mohammed RA, Martin SG, Gill MS, et al. Improved methods of detection of lymphovascular invasion demonstrate that it is the predominant method of vascular invasion in breast cancer and has important clinical consequences. Am J Surg Pathol 2007;31: 1825-1833.

30 Fox SB, Leek RD, Weekes MP, et al. Quantitation and prognostic value of breast cancer angiogenesis: comparison of microvessel density, Chalkley count, and computer image analysis. J Pathol 1995;177:275-283.

31 Hansen S, Sørensen FB, Vach W, et al. Microvessel density compared with the Chalkley count in a prognostic study of angiogenesis in breast cancer patients. Histopathology 2004;44:428-436.

32 Zehnder JL, Hirai K, Shatsky M, et al. The cell adhesion molecule CD31 is phosphorylated after cell activation. Down-regulation of CD31 in activated T lymphocytes. J Biol Chem 1992;267:5243-5249.

33 Podgrabinska S, Braun P, Velasco P, et al. Molecular characterization of lymphatic endothelial cells. Proc Natl Acad Sci USA 2002;99:16069-16074.

34 Fiedler U, Christian S, Koidl S, et al. The sialomucin CD34 is a marker of lymphatic endothelial cells in human tumors. Am J Pathol 2006;168:1045-1053.

35 Evans AJ, Rakha EA, Pinder SE, et al. Basal phenotype: a powerful prognostic factor in small screen-detected invasive breast cancer with long-term follow-up. J Med Screen 2007;14:210-214.

36 Rakha EA, Reis-Filho JS, Ellis IO. Basal-like breast cancer: a critical review. J Clin Oncol 2008;26: 2568-2581.

37 Rakha EA, Reis-Filho JS, Ellis IO. Impact of basal-like breast carcinoma determination for a more specific therapy. Pathobiology 2008;75:95-103.

38 Marks A, Sutherland DR, Bailey D, et al. Characterization and distribution of an oncofetal antigen (M2A antigen) expressed on testicular germ cell tumours. Br J Cancer 1999;80:569-578.

39 Arigami T, Natsugoe S, Uenosono Y, et al. Lymphatic invasion using D2-40 monoclonal antibody and its relationship to lymph node micrometastasis in pNo gastric cancer. Br J Cancer 2005;93:688-693.
40 Bono P, Wasenius VM, Heikkilä $\mathrm{P}$, et al. High LYVE-1positive lymphatic vessel numbers are associated with poor outcome in breast cancer. Clin Cancer Res 2004;10:7144-7149.

41 Kato T, Prevo R, Steers G, et al. A quantitative analysis of lymphatic vessels in human breast cancer, based on LYVE-1 immunoreactivity. Br J Cancer 2005;93: 1168-1174.

42 Ingeholm P, Pedersen L, Holck S. Quantification of microvessel density of breast carcinoma: an assessment of the inter- and intraobserver variation. Breast 1999;8:251-256.

43 Obermair A, Kurz C, Czerwenka K, et al. Microvessel density and vessel invasion in lymph-node-negative breast cancer: effect on recurrence-free survival. Int J Cancer 1995;62:126-131.

44 Tsutsui S, Kume M, Era S. Prognostic value of microvessel density in invasive ductal carcinoma of the breast. Breast Cancer 2003;10:312-319.

45 Costello P, McCann A, Carney DN, et al. Prognostic significance of microvessel density in lymph node negative breast carcinoma. Hum Pathol 1995;26: 1181-1184.

46 Medri L, Nanni O, Volpi A, et al. Tumor microvessel density and prognosis in node-negative breast cancer. Int J Cancer 2000;89:74-80.

47 Pàez-Ribes M, Allen E, Hudock J, et al. Antiangiogenic therapy elicits malignant progression of tumors to increased local invasion and distant metastasis. Cancer Cell 2009;15:220-231.

48 Ebos JM, Lee CR, Cruz-Munoz W, et al. Accelerated metastasis after short-term treatment with a potent inhibitor of tumor angiogenesis. Cancer Cell 2009; 15:232-239.

49 Rodríguez-Pinilla SM, Sarrió D, Honrado E, et al. Prognostic significance of basal-like phenotype and fascin expression in node-negative invasive breast carcinomas. Clin Cancer Res 2006;12:1533-1539.

50 Fulford LG, Reis-Filho JS, Ryder K, et al. Basal-like grade III invasive ductal carcinoma of the breast: patterns of metastasis and long-term survival. Breast Cancer Res 2007;9:R4. 